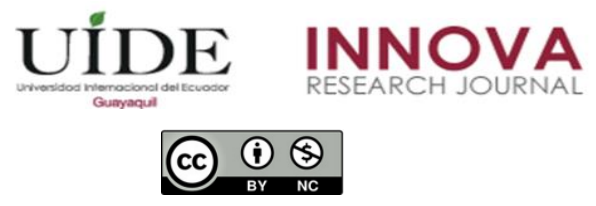

INNOVA Research Journal, ISSN 2477-9024

(Septiembre-Diciembre 2020). Vol. 5, No.3 pp. 164-181

DOI: https://doi.org/10.33890/innova.v5.n3.2020.1391

URL: http://revistas.uide.edu.ec/index.php/innova/index

Correo: innova@uide.edu.ec

\title{
Gamificación como técnica didáctica en el aprendizaje de las Ciencias Naturales
}

\section{Gamification as a teaching technique in the learning of Natural Sciences}

Angélica Janeth Mallitasig Sangucho

https://orcid.org/0000-0002-1659-8751

Pontifica Universidad Católica del Ecuador Sede Ambato, Ecuador

Teresa Milena Freire Aillón

https://orcid.org/0000-0003-2324-6495

Pontifica Universidad Católica del Ecuador Sede Ambato, Ecuador

Autor para correspondencia: yangevet@ hotmail.com; tfreire@ pucesa.edu.ec

Fecha de recepción: 03 de abril del 2020 - Fecha de aceptación: 06 de agosto del 2020

\section{Resumen}

Hacer de la educación una actividad lúdica que motive al estudiante a construir su propio aprendizaje es un reto para la comunidad educativa, sin embargo, la gamificación es una nueva técnica que en el contexto educativo mejora el aprendizaje significativo en cualquier campo del conocimiento. Por consiguiente, el presente trabajo de investigación demuestra los beneficios teóricos de la gamificación en un proceso real de aprendizaje, el objetivo es medir el logro de aprendizaje en Ciencias Naturales de los jóvenes de noveno año de la "Escuela de Educación Básica Naciones Unidas"; antes y después de la utilización de herramientas Kahoot y Plickers como parte de la gamificación, para lo cual se aplicó la Escala de Estrategias de Aprendizaje ACRA calificada en escala de Likert. Mediante análisis estadísticos se determinó que existe un considerable aumento de las estrategias de aprendizaje de los estudiantes en Ciencias Naturales, así al inicio la media global de la prueba fue de 2,3 puntos y al finalizar el programa de 6 semanas el puntaje global fue de 3,5 de una escala de 4; además mediante la prueba T-student se puede concluir que la gamificación si influye positivamente en el proceso de aprendizaje de los estudiantes de dicha institución educativa. Como conclusión, la gamificación es una técnica pedagógica innovadora que combina los elementos del juego para que el estudiante interioriza el conocimiento y viva el aprendizaje como una experiencia positiva y satisfactoria.

Palabras claves: gamificación; aprendizaje significativo; Kahoot; Plickers; ciencias naturales

\begin{abstract}
Making education a playful activity that motivates the student to build their own learning is a challenge for the educational community, however, gamification is a new technique that in the educational context improves meaningful learning in any field of knowledge. Therefore, the present research work demonstrates the theoretical benefits of gamification in a real learning process, the objective is to measure the achievement of learning in Natural Sciences in the ninth-
\end{abstract}


year young boys of the School of Basic Education United Nations; before and after the use Kahoot and Plickers as part of the gamification, for which the ACRA Learning Strategies Scale rated on a Likert scale was applied. Through statistical analysis it was determined that there is a considerable increase in the learning strategies of students in Natural Sciences, so at the beginning the global mean of the test was 2.3 points and at the end of the week program the overall score was 3,5 ; In addition, through the T-student test, it can be concluded that gamification does positively influence the learning process of the students of said educational institute. In conclusion, gamification is an innovative pedagogical technique that combines the elements of the game so that the student internalizes knowledge and lives learning as a positive and satisfying experience.

Key Words: gamification; meaningful learning; Kahoot; Plickers; natural sciences

\section{Introducción}

El proceso de aprendizaje puede tornarse aburrido y monótono para el estudiante, lo que provoca desinterés por aprender (Beltrán Morales, 2017). Por ello, en un entorno en constante cambios y evolución tecnológica, es importante transformar el rol de los docentes, estudiantes y padres de familia en el arte de enseñar y aprender. (Hurtado y otros, 2018).

En el proceso pedagógico es indispensable que el docente use y cree nuevos y diferentes modelos, metodologías, técnicas en el aula que aporten a la educación y que sirvan para cubrir las necesidades de los alumnos a fin de lograr que el aprendizaje sea significativo (Romero Zegarra, 2016). Tal motivo ha generado que se popularice el uso de nuevas plataformas de aprendizaje en donde los estudiantes no solo interactúan con sus compañeros, sino se entrelazan con material en distintos formatos como son videos, audios, juegos; respondiendo a la teoría del aprendizaje constructivista donde el estudiante es un sujeto activo que debe formar su conocimiento a través de la interacción con su entorno (Menendez y otros, 2018).

Por otra parte, el aprendizaje de las ciencias naturales pretende la formación basado en una actitud científica de los estudiantes que le permita entender la ciencia como una búsqueda lógica y sistemática para entender la realidad (Adúriz Bravo y otros, 2011). Debe ser apoyado en estrategias didácticas innovadoras, alternativas, de indagación, formulación, solución de problemas, en donde el principal elemento sea el estudiante, de esta manera se espera cambiar el modelo tradicional de estudio (Torres y otros, 2013).

Una posibilidad para ayudar al desarrollo del aprendizaje es la gamificación, que es una de las técnicas que utiliza el juego como recurso didáctico, cuya aplicación ayuda a desarrollar destrezas y habilidades, genera procesos de retroalimentación entre compañeros de estudio, lo que se puede comprobar con el progreso de los conocimientos en solución de problemas, convirtiéndose las actividades difíciles en procesos más sencillos (Rodríguez y Elías, 2018).

Sánchez y otros (2018) en su investigación hacen énfasis en el desarrollo de la gamificación basada en las TIC, para que los estudiantes de Odontología de la UDLA logren un fortalecimiento de su léxico adquiriendo nuevo vocabulario del idioma inglés, utilizando la metodología de recopilación de información bibliográfica y documental, trabajando con dos grupos de estudiantes: grupo experimental (uso de gamificación) y grupo de control (uso de tratamiento). Dando como resultado que los alumnos pertenecientes al grupo experimental 
lograron mayor desarrollo en su competencia léxica, por otro lado, en los estudiantes del grupo de control no se evidenciaron diferencias significativas en el aprendizaje.

Para López García (2016) la enseñanza de artísticas en los alumnos toma mucho tiempo y puede ser un proceso complicado, pero al introducir las TIC, en este caso la herramienta Plickers, se ha visto una solución de evaluar a los estudiantes, de manera instantánea y distinta a una clase tradicional, cuyo fin es el de motivar y desarrollar habilidades dentro de la asignatura de música.

En la investigación de González (2016) realiza varias actividades con un grupo de estudiantes en torno a la gamificación la cual consiste en la utilización de ciertos juegos para cambiar el comportamiento de los individuos, de manera que éstos mejoren su motivación y así alcanzar los objetivos deseados. Puesto que hoy en día la juventud está sometido a múltiples estímulos externos los cuales provocan distracción y hacen que el aprendizaje se torne monótono, obligado y aburrido.

Por ende, el objetivo de éste estudio es medir, comparar, logros adquiridos antes y después de la implementación de estrategias de gamificación con los alumnos de la institución educativa a estudiar. Es importante porque en el marco del aprendizaje significativo, se analiza el impacto de la gamificación como técnica didáctica a través de la inclusión de herramientas; es útil para la innovación de nuevas propuestas educativas que apoyen el aprendizaje constructivista en un entorno donde las tecnologías de la información y comunicación son parte del diario vivir de los estudiantes. El desarrollo de este es factible por los amplios fundamentos teóricos acerca de la técnica de la gamificación en diversos repositorios online; además la disponibilidad de la unidad de estudio para colaborar abiertamente con dicho caso.

\section{Marco Teórico}

La gamificación tiene como fin, lograr que una actividad educativa no lúdica se vuelva algo interesante para el estudiante. Esta nueva técnica anima a los individuos a realizar tareas que son aburridas, con el objetivo de conseguir logros o recompensas (Quesada, 2014). Existen diversas conceptualizaciones de la gamificación, pero (Gallego y otros, 2014) realizan una compilación y nos muestra una definición concisa del término. He aquí sus propias palabras:

El uso de estrategias, modelos, dinámicas, mecánicas y elementos propios de los juegos en contextos ajenos a éstos, con el propósito de transmitir un mensaje o unos contenidos o de cambiar un comportamiento, a través de una experiencia lúdica que propicie la motivación, la implicación y la diversión. (p. 2)

De manera sintetizada, la finalidad de la gamificación es buscar la motivación del individuo como base para que cumpla un objetivo en cualquier ámbito (empresarial, educativo, salud, deporte, entre otros) que no sea el juego pero que combina los elementos de este para crear situaciones lúdicas.

El origen de la gamificación se basó en la Teoría de Flujo propuesta por Mihály Csíkszentmihályi en 1975, la cual explica que es un estado dinámico de experiencia óptima que produce placer por sí misma, que las personas sienten cuando están intensamente implicadas en 
lo que están haciendo y que les resulta divertido hacer, enfocándose en el camino mas no en el objetivo que se desea alcanzar (Mesurado, 2010). Esta teoría se puede comprender fácilmente con los videojuegos, cuando el jugador ha conocido la mecánica y la lógica se sumerge en el mismo y no considera el tiempo debido al placer que le genera la experiencia (Ciucci, 2016). De ahí que la gamificación se relaciona al mundo de los videojuegos donde el individuo es un sujeto activo, predispuesto a realizar una actividad que le motive, le llame la atención, y se comprometa con su propio aprendizaje, aumente la diversión y disminuya la sensación de esfuerzo (Pisonero, 2018).

A pesar de que el termino gamificación o "gamification" (en inglés) ha existido y ha sido empleado desde hace tiempo, especialmente en el contexto empresarial, su autoría no está bien definida. Autores como Werbach y Hunter dedican el origen a Nick Pelling quien fundó en 2002 una pequeña consultora de negocios, Conundra, que consistía en poner en práctica mecánicas y conceptos presentes en los juegos, procesos, productos y servicios de sus clientes. Por otro lado, los autores Deterling, Dixon, Khaled y Nacke otorgan la autoría a escritores de blog a lo largo del 2008, el primero pertenece a Terril quien utilizó el término "gameification" (posteriormente, aceptado como gamification) para referirse que las empresas buscaban, mediante el juego, el desarrollo de páginas y servicios para elevar su audiencia. El segundo, pertenece a Currier para hacer referencia a la importancia que alcanzaron los juegos en los medios de comunicación, en las décadas de los 40 y 50, para influenciar con mensajes persuasivos o publicitarios (Novillo y otros, 2018).

Sin embargo, el hecho de usar un juego, incluso como técnica para conseguir un fin educativo, no constituye por sí solo gamificación como lo sugiere Cortizo y otros (como se citó en Morales, 2013). Por ende, la gamificación se basa en tres fundamentos propuestos por Werbach:

- Las dinámicas. - son el concepto, la estructura implícita del juego.

- Las mecánicas. - son los procesos que provocan el desarrollo del juego

- Los componentes. - son las implementaciones específicas de las dinámicas y mecánicas: avatares, insignias, puntos colecciones, rankings, niveles, equipos, entre otros. Werbach: (como se citó en Ortiz y otros, 2018).

En consecuencia, la relación recíproca por parte de éstos tres fundamentos en cuanto a la acción, crea una verdadera actividad lúdica guiada hacia el conocimiento. Además, para una comprensión más amplia cada fundamento contiene diversos elementos, como se muestra en la tabla 1:

\section{Tabla 1}

Clasificación de los elementos de gamificación

\begin{tabular}{lll}
\hline \multicolumn{1}{c}{ Grupo } & \multicolumn{1}{c}{ Definición } & \multicolumn{1}{c}{ Elementos del grupo } \\
\hline Dinámicas & Ponen en prácticas las mecánicas del juego tiene & Limitaciones \\
& relación con las motivaciones y objetivos del & Emociones \\
& jugador. & Narrativa \\
& & Relativa \\
\hline
\end{tabular}




\begin{tabular}{lll}
\hline \multicolumn{1}{c}{ Grupo } & \multicolumn{1}{c}{ Definición } & \multicolumn{1}{c}{ Elementos del grupo } \\
\hline Mecánicas & Componentes o elementos básicos del juego & Turnos \\
& & Feedback \\
& & Oportunidades \\
& Retos \\
& & Competición. \\
\hline Componentes & Materiales y herramientas de diseño para el & Puntos \\
& implementar el juego. & Niveles \\
& & Avatares \\
& & Equipos \\
& & Logros \\
\hline
\end{tabular}

Fuente: Mora, Calderón, y Rubio (2018)

\section{Gamificación en la educación}

En el contexto educativo, Pilar Rivero (2017) conceptualiza la gamificación como: "la introducción de mecánicas y dinámicas de juego en el aula y en cualquier entorno, en principio, ajeno al juego "Pilar Rivero (como se citó por Navarrete García, 2017). Esta técnica fomenta el aprendizaje significativo al brindar una experiencia enriquecedora y atractiva a las actividades en el aula de clase, motivar a los estudiantes a través de mecanismos de motivación intrínsecos y extrínsecos (Romero Zegarra, 2016), (Barragán Piña, y otros, 2015).

La gamificación educativa, en la actualidad es una tendencia que supone la fusión del concepto de ludificación y aprendizaje (Marín, 2015); se constituye en una actividad de aprendizaje más, que al igual que otras técnicas pedagógicas tiene como finalidad que el estudiante capte el conocimiento y, posteriormente, pueda poner en práctica. Sin embargo, esta técnica en específico tiene un diseño particular que aprovecha las emociones y comportamientos de los jugadores para aumentar la unión, integración y la motivación por el contenido de cualquier área del conocimiento conduciendo al estudiante a construir su aprendizaje por sí mismo y no por obligación (Foncubierta y Rodríguez, 2014).

En este ámbito y en la línea del Constructivismo Smith (2011) señala que la gamificación debe cubrir dos objetivos básicos:

- Hacer más transparente el progreso del aprendizaje, de forma que el alumno tome consciencia del paso del tiempo y cómo su conocimiento se va incrementando.

- Fomentar el autoaprendizaje haciendo que el alumno deje de ser un receptor pasivo y pase a ser un valor activo en el aprendizaje Smith (como se cita en Parra y Torres, 2018, pág. 162).

En consecuencia, el papel del profesor es facilitar el aprendizaje para lo cual en referencia a los elementos del juego (tabla 1) también deberá construir las reglas del juego porque son detalles a los que los estudiantes están acostumbrados y enriquecen la experiencia, tales como: límite de tiempo, puntuaciones, medallas, logros, superación de niveles, pero también colaboración, trabajo en equipo, búsqueda de soluciones, estrategia y planificación, entre otros (Barragán Piña, y otros, 2015). 
$\mathrm{Al}$ parecer es una técnica fácil de implementar en el aula por la compatibilidad entre el grupo objetivo y su relación con los fundamentos de la gamificación (el juego), sin embargo; presenta algunos retos que se deben superar para no crear un proceso equivocado semejante a la cercanía entre el amor y el odio (Parente, 2016). Parente (2016) señala cuatro casos peligrosos que se deben considerar para un adecuado uso de la gamificación, los cuales se describen a continuación:

1. No son juegos en el aula de clase.

2. Implica a la comunidad educativa.

3. Requiere un equipo multidisciplinar.

4. Equilibrio del proceso (p. 18-19).

1.- El objetivo del juego es preferencialmente la coordinación motora, el de la gamificación es crear condiciones lúdicas para el aprendizaje. Dicho de otra manera, uno de los fundamentos de la gamificación es la mecánica del juego para crear retos en el estudiante y motivarlo. Sin embargo, la acción no se queda ahí, sino que es un proceso más avanzado encaminado a aprender, en concordancia con Werbach y Hunter (2012) que mencionan que: "la gamificación consiste en el uso de elementos de juegos y técnicas de diseño de juegos en contextos que no lúdicos” Werbach y Hunter (como se citó por Biel y García, 2015).

2.- El propósito es crear un ambiente responsable con la implementación de la gamificación donde el profesor no sea el único protagonista, en otras palabras, el docente no es el único que debe proponer dicha técnica, sino que la institución educativa proponga la estrategia basada en principios para que cualquier docente la pueda implementar y crear una estructura compleja que sea capaz de sustentar la correcta gestión de la gamificación.

3.- La gamificación dentro del aula, debe ser un trabajo conjunto con el docente, para que los resultados sean más positivos, el diseñador del videojuego y neuropsicólogos que junten sus conocimientos para que la estrategia y el conocimiento estén acorde a los estudiantes objetivo. $\mathrm{Al}$ igual que el punto anterior, debe existir la comprensión e información de la gamificación por toda la comunidad educativa.

4.- Debe existir un equilibrio entre las habilidades y conocimientos previos del estudiante con relación a los retos que se plantean en la gamificación, de tal manera que los conocimientos no sobrepasen a los retos porque si no se convertiría en una actividad aburrida o, al contrario, que los retos sean superiores a los conocimientos porque generaría angustia en los estudiantes. Debe ser un proceso paulatino, de acuerdo hasta qué punto se debe abarcar el saber.

Otro aspecto para considerar en la gamificación es que, en la actualidad, el avance tecnológico y el uso de ésta por parte de los estudiantes van al mismo ritmo, es decir, las nuevas generaciones conviven con la tecnología y se convierten parte de su diario vivir. En éste sentido, los nuevos aprendizajes plantean la utilización de las herramientas web 2.0 en un entorno didáctico (Zambrano Izquierdo, Gómez Zermeño \& Guerrero Roldan,2016).

Con respecto a lo anterior, toda actividad de enseñanza-aprendizaje necesita fomentar la disciplina en el aula. Para ello (Zambrano Izquierdo , Gomez Zermeño, \& Guerrero Roldan, 
2016) plantean los siguientes principios para la gamificación en un entorno tecnológico (basados en Zichermann y Cunningham, 2011; Observatorio de innovación, 2015; Marín, 2015; NMC, 2015):

- El establecimiento de un sistema de progresión que permita al aprendiz identificar el logro de sus aprendizajes.

- La clasificación o posicionamiento es otro elemento en la gamificación, ya que permite al estudiante reflexionar acerca de su participación activa en la interacción con el recurso educativa.

- El diseño de la experiencia de aprendizaje debe ofrecer un enfoque basado en metas, reglas, retos y puntos a ganar al concluir una tarea.

- Ofrecer un sistema de insignias, logros y recompensas que promuevan la motivación del aprendiz.

Retomando la importancia de las TICs en la gamificación, hay que decir que la Web 2.0 contiene aplicaciones que animan la interacción entre el usuario y la internet (Pérez Salazar, 2011). En consecuencia, el desarrollo de herramientas tecnológicas apoya la técnica de la gamificación por medio de la creación de juegos digitales tales como Kahoot y Plickers que han sido utilizados en la presente investigación.

Kahoot es una herramienta gratuita y libre que sirve para la retroalimentación de manera activa o para la evaluación mediante la creación de cuestionarios en línea que pueden contener imágenes o videos y que pueden ser respondidos por los estudiantes a través de sus dispositivos móviles personales. Al mismo tiempo crea una clase divertida con un ambiente de competición sana, desarrolla el aprendizaje en conjunto, crea una mayor incidencia en el aspecto pedagógico, pone a prueba el conocimiento a la vez que ayuda a la evaluación y retroalimentación (Biel y García, 2015); (Gonzales, 2016); (Carrión, 2017) ; (Torres y Romero, 2018).

Otra herramienta tecnológica es la denominada Plickers; es digital y gratuita, disponible para $i O S$ y Android, contribuye con la evaluación de los conocimientos, muestra resultados en el momento preciso, con la facilidad de visualizar las calificaciones en términos relativos. Al ser una forma de evaluación cuantitativa facilita el análisis del logro alcanzado, así como también ayuda a resolver las falencias de conocimiento que pueda existir en el estudiante o grupo de estudiantes (López, 2016).

Lo más imprescindible al utilizar cualquier herramienta para la gamificación en el aula, es considerar qué saberes y habilidades se necesita desarrollar en los estudiantes, hay que tener presente que la intención es el aprendizaje constructivo y no confundir la situación con la utilización de dispositivos y herramientas tecnológicas con el hecho de que son aspectos familiares con los estudiantes.

\section{Metodología}




\section{Enfoque}

La presente investigación aplicada experimental se realiza en dos tiempos, con la finalidad de controlar la variable logro del aprendizaje, es decir, antes y después de implementar la variable gamificación mediante distintas aplicaciones. Adicionalmente, la investigación es de tipo descriptivo porque se detalle el fenómeno a estudiar, posee un enfoque mixto donde se mide el logro del aprendizaje en Ciencias Naturales de manera cuantitativa (mediante pruebas estadísticas) y cualitativa (explicando la significancia de los valores numéricos).

\section{Unidad de análisis}

El estudio se llevó a cabo con la participación de 30 estudiantes de noveno año de educación básica de la Escuela de Educación Básica Naciones Unidas del Cantón Saquisilí (Cotopaxi-Ecuador), considerados como la fuente primaria para la obtención de datos. El periodo de estudio y recolección de datos fue el segundo quimestre, tercer parcial del año lectivo 20192020 .

\section{Técnicas para recoger datos}

La recopilación de los datos se lo realiza a través de la Escala de Estrategias de Aprendizaje ACRA (Adquisición, Codificación, Recuperación y Apoyo) desarrollado y definido por primera vez por Román y Gallego (1994). La finalidad de ACRA es conocer el tipo de procesamiento que el alumno desarrolla con el nuevo conocimiento impartido, ya sea éste, superficial o significativo (Román y Gallego, 2008, pág. 8). ACRA esta estructura por cuatro escalas o dimensiones que se detallan en la tabla 2:

Tabla 2

Estructura de la Escala de Estrategias de Aprendizaje ACRA

\begin{tabular}{|c|c|c|}
\hline Escala o dimensión & Estrategias & $N^{0}$ ítems \\
\hline $\begin{array}{l}\text { Escala I } \\
\text { Adquisición }\end{array}$ & $\begin{array}{l}\text { - Estrategias atencionales que incluye la exploración y la } \\
\text { fragmentación } \\
\text { - Estrategia de repetición }\end{array}$ & 20 ítems \\
\hline $\begin{array}{l}\text { Escala II } \\
\text { Codificación }\end{array}$ & $\begin{array}{l}\text { - Estrategia de menotecnización con el uso de acrósticos, rimas, } \\
\text { muletillas entre otros } \\
\text { Estrategia de elaboración que incluye relaciones, imágenes, } \\
\text { metáforas, analogías, aplicaciones de los contenidos, auto preguntas } \\
\text { y parafraseado } \\
\text { Estrategia de organización que incluye el agrupamiento, secuencias, } \\
\text { mapas, diagramas }\end{array}$ & 46 ítems \\
\hline $\begin{array}{l}\text { Escala III } \\
\text { Recuperación }\end{array}$ & $\begin{array}{l}\text { - Estrategia de búsqueda de codificaciones e indicios } \\
\text { - Estrategia de generación de respuestas de planificación y respuesta } \\
\text { escrita }\end{array}$ & 18 ítems \\
\hline
\end{tabular}




\begin{tabular}{llll}
\hline \multicolumn{1}{l}{ Escala o dimensión } & \multicolumn{1}{c}{ Estrategias } & $\mathbf{N}^{\mathbf{0}}$ ítems \\
\hline $\begin{array}{l}\text { Escala IV } \\
\text { Apoyo }\end{array}$ & $\bullet$ & $\begin{array}{l}\text { Estrategias metacognitivas que incluye el autoconocimiento y el } \\
\text { automanejo }\end{array}$ & 35 ítems \\
& $\bullet$ & $\begin{array}{l}\text { Estrategias socioafectivas que evalúa las activas, sociales y } \\
\text { motivacionales }\end{array}$ \\
& &
\end{tabular}

Fuente: Román Sánchez y Gallego Rico (2008)

Adicionalmente, con el fin de medir el grado de satisfacción de la técnica aplicada a los estudiantes de la Escuela de Educación Básica Naciones Unidas se desarrolló una encuesta de satisfacción con 10 ítems denominados desde P1 hasta P10 que se califican con escala de Likert (Anexo 1). A continuación, el significado de cada pregunta con respecto a la gamificación:

Tabla 3

Preguntas de la encuesta de satisfacción

\begin{tabular}{ll}
\hline Pregunta & Finalidad \\
\hline P1 & Adecuación \\
P2 & Motivación \\
P3 & Interacción y entretenimiento \\
P4 & Mejor aprendizaje \\
P5 & Interacción \\
P6 & Innovación \\
P7 & Utilidad \\
P8 & Colaboración \\
P9 & Comprensión y retención de información \\
P10 & Herramienta de gamificación \\
\hline
\end{tabular}

Fuente: Elaboración propia

\section{Procesamiento de análisis}

Para medir el impacto de la gamificación en el nivel de logro de aprendizaje de la asignatura de Ciencias Naturales, se utilizó Escala de Estrategias de Aprendizaje ACRA para lo cual primeramente se comprobó su validez y confiabilidad mediante el estadístico Alpha de Cronbach. Este expresa el grado de homogeneidad de los ítems, con la intención de que juntos tengan significancia y se relacionen la variable de estudio, de ésta manera los datos recolectados serán considerados oportunos y confiables (Quero Virla, 2010). Al correr dicho coeficiente proporcionó un valor de 0,972 de los 119 ítems del instrumento lo que indica una buena consistencia interna.

La aplicación del instrumento se realizó en dos tiempos; antes y después de la implementación de la gamificación mediante herramientas como Kahoot y Plickers. Con respecto a los contenidos de Ciencias Naturales que se evaluaron, estos se refieren a la sexta unidad titulada "Nuestro Planeta" planificada cubrirse en seis semanas de trabajo. En la tabla 4 se muestra los contenidos y actividades desarrolladas durante la ejecución de la propuesta. 


\section{Tabla 4}

Contenidos de la clase tratados durante la ejecución de la técnica de gamificación

\begin{tabular}{lll}
\hline \multicolumn{1}{c}{ Semanas de trabajo } & \multicolumn{1}{c}{ Contenidos } & \multicolumn{1}{c}{ Técnicas y actividades } \\
\hline $\begin{array}{l}\text { Primera Semana } \\
\text { Segunda Semana } \\
\text { Tercera Semana }\end{array}$ & $\begin{array}{l}\text { Biomas de la tierra } \\
\text { Biomas terrestres } \\
\text { Biomas acuáticos y biodiversidad- } \\
\text { conservación }\end{array}$ & $\begin{array}{l}\text { Clase magistral } \\
\text { Clase gamificada con Kahoot } \\
\text { Clase gamificada con Plickers }\end{array}$ \\
$\begin{array}{l}\text { Biodiversidad del Ecuador - } \\
\text { regiones del Ecuador }\end{array}$ & Clase gamificada con Kahoot \\
Suinta semana & $\begin{array}{l}\text { Ecosistemas del Ecuador } \\
\text { Presentación del proyecto final }\end{array}$ & $\begin{array}{l}\text { Clase gamificada con Plickers } \\
\text { Desarrollo del proyecto } \\
\text { biodiversidad de las regiones del } \\
\text { ecuador }\end{array}$ \\
\hline
\end{tabular}

Fuente: Elaboración propia

Adicionalmente, para demostrar que existen discrepancias importantes en la asimilación de las Ciencias Naturales con la técnica de gamificación se plantean la siguiente hipótesis:

Ho: La técnica de gamificación no influye en el proceso de aprendizaje de los estudiantes de la materia de Ciencias Naturales en la Escuela de Educación Básica Naciones Unidas

H1: La técnica de gamificación si influye en el proceso de aprendizaje de los estudiantes de la materia de Ciencias Naturales en la Escuela de Educación Básica Naciones Unidas.

Por otra parte, para comprobar la validez de la encuesta de satisfacción de los estudiantes también se corrió el estadístico Alpha de Cronbach; cuyo resultado fue 0,908 lo cual quiere decir que hay una muy buena confiabilidad.

\section{Resultados}

Se efectuó el análisis del estudio descriptivo de los resultados del instrumento aplicado antes y después de la implementación de la técnica de gamificación. Hay que considerar que la puntuación del test de ACRA está en escala de Likert del 1 al 4; donde 1(Nunca o casi nunca) significa poca o nula estrategia de aprendizaje y 4 (Siempre y casi siempre) significa altas estrategias de aprendizaje.

\section{Tabla 5}

Estadísticos descriptivos del test ACRA

\begin{tabular}{llllll}
\hline & Estadísticos & \multicolumn{1}{c}{ Escala I } & \multicolumn{1}{c}{ Escala II } & \multicolumn{1}{c}{ Escala III } & Escala IV \\
\hline Antes & Muestra & 30 & 30 & 30 & 30 \\
& Media & 2,3333 & 2,2051 & 2,3463 & 2,5152 \\
Mediana & 2,325 & 2,1522 & 2,3056 & 2,3571 \\
& Desviación & 0,50996 & 0,48783 & 0,60007 & 0,74899 \\
estándar & & & & \\
Mínimo & 1,35 & 1,28 & 1,28 & 1,26 \\
& Máximo & 3,35 & 3,15 & 3,78 & 4,11 \\
\hline
\end{tabular}




\begin{tabular}{llllll}
\hline & Estadísticos & \multicolumn{1}{c}{ Escala I } & \multicolumn{1}{c}{ Escala II } & Escala III & Escala IV \\
\hline Después & Muestra & 30 & 30 & 30 & 30 \\
& Media & 3,5083 & 3,3014 & 3,5463 & 3,4781 \\
& Mediana & 3,7 & 3,413 & 3,5556 & 3,6571 \\
& Desviación & 0,52933 & 0,23272 & 0,23932 & 0,39929 \\
estándar & & & & \\
& Mínimo & 2,15 & 2,41 & 3 & 2,29 \\
& Máximo & 4 & 3,48 & 3,83 & 3,77 \\
\hline
\end{tabular}

Fuente: Elaboración propia

En escala I la puntuación media antes de la implementar la técnica de gamificación es de 2,3 y después es de 3,5; en la escala II la puntuación media antes es de 2,2 y después de 3,3; en la escala III la media antes es de 2,3 y después de 3,5; y en la escala IV la media antes es de 2,5 y después de 3,4. Lo que se evidencia que, efectivamente las actividades planificadas como la técnica de gamificación aumentó el interés de los estudiantes a lo largo del proceso de aprendizaje en todas las dimensiones, es decir, que la información se codificó adecuadamente para convertirse en conocimiento en cada estudiante. La introducción de nuevas pedagogías hace aún más interesante las clases, despertando la curiosidad y creando mayor disposición a la comprensión.

En todas las escalas de ACRA ha incrementado el aprendizaje con la gamificación, como se observa en la figura 1:

\section{Figura 1}

La puntuación media en la prueba ACRA

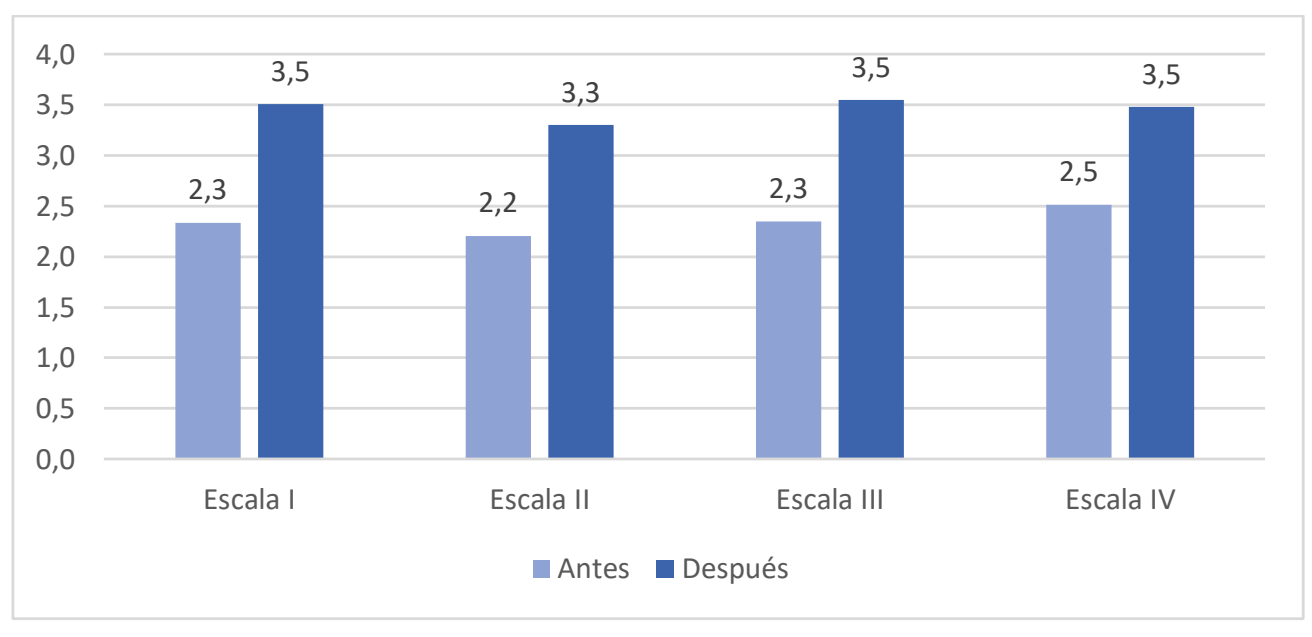

La puntuación media en la prueba ACRA antes de la técnica de la gamificación no supera los 2,5 puntos en cada escala, luego con la implementación de las herramientas Kahoot y Plickers la puntuación media incrementa aproximadamente un punto en todas las escalas. 
El incremento de las estrategias de aprendizaje es homogéneo en todas las dimensiones que presenta el instrumento, este efecto nos muestra que la gamificación es una técnica completa, que causa ventajas en la adquisición, codificación, recuperación y apoyo; lo que evita brechas en el aprendizaje.

El incremento del aprendizaje de Ciencias Naturales de aproximadamente un punto en cada escala de la prueba ACRA y para comprobar que este incremento es significativo se corrió la prueba de hipótesis T-student para muestras relacionadas como es el caso de que al mismo grupo que se evaluó con la prueba ACRA en dos momentos. Los resultados se muestran en la tabla 6:

\section{Tabla 6}

Estadístico T-student para muestras relacionadas

\begin{tabular}{|c|c|c|c|c|c|c|c|c|}
\hline \multirow{3}{*}{$\begin{array}{l}\text { Antes - } \\
\text { Después }\end{array}$} & \multicolumn{5}{|c|}{ Diferencias emparejadas } & \multirow{3}{*}{$\mathbf{t}$} & \multirow{3}{*}{ gl } & \multirow{3}{*}{$\begin{array}{c}\text { Sig. } \\
\text { (bilateral }\end{array}$} \\
\hline & \multirow{2}{*}{ Media } & \multirow{2}{*}{$\begin{array}{l}\text { Desviación } \\
\text { estándar }\end{array}$} & \multirow{2}{*}{$\begin{array}{l}\text { Media de } \\
\text { error } \\
\text { estándar }\end{array}$} & \multicolumn{2}{|c|}{$\begin{array}{l}95 \% \text { de intervalo de } \\
\text { confianza de la diferencia }\end{array}$} & & & \\
\hline & & & & Inferior & Superior & & & \\
\hline Escala I & $-1,17$ & 0,75221 & 0,13733 & $-1,45588$ & $-0,89412$ & $-8,556$ & 29 & 0 \\
\hline Escala II & $-1,096$ & 0,48827 & 0,08914 & $-1,2787$ & $-0,91406$ & $-12,29$ & 29 & 0 \\
\hline Escala III & $-1,2$ & 0,66147 & 0,12077 & $-1,447$ & $-0,953$ & $-9,936$ & 29 & 0 \\
\hline Escala IV & $-0,962$ & 0,97738 & 0,17845 & $-1,32782$ & $-0,5979$ & $-5,396$ & 29 & 0 \\
\hline
\end{tabular}

Fuente: Elaboración propia

En todas las escalas se aprecia, que la prueba estadística T-student para muestras relacionas es significativa, es decir que el sig es menor o igual a 0,05 con una confiabilidad del $95 \%$. En este caso en todas las escalas se tiene un valor del sig de cero, lo que quiere decir que existen diferencias significativas después de aplicar la técnica de gamificación en los estudiantes de noveno año de educación básica de la Escuela de Educación Básica Naciones Unidas.

Entonces, el aprendizaje logrado con la utilización de la gamificación no se puede considerar como un recurso vago, por el contrario, los efectos de su utilización pueden crear grandes e importantes cambios transversales en la manera de enseñar y la profundidad de la captación del mensaje que el maestro imparte a sus educandos.

Con referencia a la satisfacción de los estudiantes al utilizar la técnica de la gamificación se analizó los primeros 9 ítems que hacen referencia a determinar el grado de satisfacción de la técnica de gamificación en el aprendizaje de las Ciencias Naturales; se tiene las siguientes respuestas: 
Tabla 7

Satisfacción con respecto a la gamificación

\begin{tabular}{lccccccccc} 
Respuestas & P1 & P2 & P3 & P4 & P5 & P6 & P7 & P8 & P9 \\
\hline A (extremadamente Satisfactorio) & 17 & 19 & 23 & 18 & 17 & 18 & 17 & 24 & 20 \\
B (muy satisfactorio) & 13 & 11 & 3 & 11 & 11 & 9 & 13 & 4 & 8 \\
C (moderadamente satisfactorio) & & & 3 & 1 & 2 & 3 & & 2 & 2 \\
D (poco satisfactorio) & & & 1 & & & & & & \\
E (nada satisfactorio) & & & & & & & & & \\
\hline Total & 30 & 30 & 30 & 30 & 30 & 30 & 30 & 30 & 30 \\
\hline
\end{tabular}

Fuente: Elaboración propia

Se aprecia que un 64,07\% de las respuestas dicen que la técnica es extremadamente satisfactoria, un 30,74\% señala que la técnica que es muy satisfactorio y un mínimo del 5,19\% dicen que la técnica de la gamificación es moderadamente satisfactoria.

Una pregunta de la encuesta (P10) estaba destina a determinar qué herramienta de gamificación que se utilizó es más interactiva para los estudiantes. Los resultados obtenidos se describen a continuación:

Tabla 8

Satisfacción en relación a la herramienta de gamificación

\begin{tabular}{ccc}
\hline Herramienta web 2.0 & Frecuencia & Porcentaje \\
\hline Kahoot & 25 & $83,3 \%$ \\
Plickers & 5 & $16,7 \%$ \\
Total & 30 & $100 \%$ \\
\hline
\end{tabular}

Fuente: Elaboración propia

Se aprecia que de los 30 estudiantes que se aplicó la técnica de gamificación a través de herramientas de gamificación, a 25 de ellos que representan el 83,3\% está más inclinado por la aplicación Kahoot para mejorar su aprendizaje y tan solo 5 que representan el $16.7 \%$ estudiantes prefieren la aplicación Plickers.

Lo que quiere decir que la técnica puesta a prueba en este estudio, tiene una interfaz amigable, compatible con la realidad tecnológica en que se desarrollan los jóvenes, además, no solo es cuestión de buscar una aplicación, si no de que las características sean acoplables a los estudiantes. En este caso, se puede afirmar que Kahoot tiene una mejor composición mecánica que Plickers.

\section{Conclusiones}

La gamificación aplicada al ámbito educativo mediante herramientas tecnológicas es una técnica didáctica eficaz, se pudo verificar que los educandos del noveno año de la "Escuela Naciones Unidas" (unidad de estudio) el aprendizaje significativo tuvo grandes avances al desarrollar el plan de clases de la unidad "Nuestro Planeta" de la asignatura de Ciencias 
Naturales. En tan solo 6 semanas de complementar la pedagogía utilizando Kahoot y Plickers, la puntuación media de la Escala de Estrategias de Aprendizaje ACRA aumentó de 2,34 a 3,45 lo que significa un incremento del $32 \%$ en el aprendizaje de los estudiantes.

Complementariamente, a través de la prueba estadística T-student para muestras relacionadas se puede concluir que la técnica didáctica de la gamificación en el aprendizaje de Ciencias Naturales influye de manera positiva en todas las escalas de la prueba ACRA, como consecuencia esta técnica sí mejora la interiorización del conocimiento del grupo de estudiantes de la institución en estudio.

Además, es correcto afirmar que los estudiantes, en su gran mayoría, consideran que la técnica de la gamificación es satisfactoria y más aceptada por los estudiantes; se concluye que algunos efectos de la gamificación en la educación son la mayor interacción, entretenimiento y motivación por las Ciencias Naturales y que el conocimiento se capta más rápido y se logra un aprendizaje a largo plazo.

Por ende, se recomienda a los pedagogos capacitarse y adoptar nuevas metodologías de enseñanza apegadas a la realidad y cualidades de los alumnos. La educación debe evolucionar conjuntamente con las personas y ser un espacio de interés y motivador para que sea más factible abrir las mentes a nuevos conceptos e implantar conocimientos, y la gamificación es una de éstas técnicas, y las herramientas presentadas en éste estudio Kahoot y Plickers tan solo son una mínima parte de lo que podemos acceder para encaminar las clases en cualquier área del conocimiento, dando como resultado un proceso enseñanza-aprendizaje eficiente y significativo.

\section{Bibliografía}

Adúriz Bravo, A., Gómez Galindo, A. A., Rodríguez Pineda, D. P., López Valentín, D. M., Jiménez Aleixandre, M. D., Izquierdo Aymerich, M., \& Sanmartí Puig, N. (2011). Las ciencias naturales en educación básica: formación de la ciudadanía para el siglo XXI. Cuauhtémoc.

Obtenido de http://www7.uc.cl/sw_educ/educacion/grecia/plano/html/pdfs/biblioteca/LIBROS/LIbroA gustin.pdf

Barragán Piña, A. J., Ceada Garrido, Y., Andújar Márquez, J. M., Irigoyen Gordo, E., Gómez Garay, V., \& Artaza Fano, F. (2015). Una propuesta para la motivación del alumnado de ingeniería mediante técnicas de gamificación. XXXVI Jornadas de Automática, (págs. 710715).

Bilbao.

Obtenido

de http://rabida.uhu.es/dspace/bitstream/handle/10272/11116/Una_propuesta_para_la_motiv acion.pdf?sequence $=5$

Barragán, A. J., Ceada, Y., Andújar, J. M., Irigoyen, E., Gómez, V., \& Artaza, F. (2015). Una propuesta para la motivación del alumnado de ingeniería mediante técnicas de gamificación. Actas de las XXXVI Jornadas de Automática, 2 - 4 de septiembre de 2015 (págs. 710 - 715). Bilbao: Comité Español de Automática de la IFAC (CEA-IFAC). Obtenido de http://rabida.uhu.es/dspace/handle/10272/11116

Beltrán Morales, J. T. (2017). E-learning y gamificación como apoyo al aprendizaje de programación. Universidad de Extremadura. Universidad de Extremadura. Obtenido de 
http://dehesa.unex.es/bitstream/handle/10662/6429/TDUEX_2017_Beltr\%c3\%a1n_Mora les.pdf?sequence $=1 \&$ isAllowed $=\mathrm{y}$

Biel, L., \& García, A. (2015). Gamificar: El uso de los elementos del juego en la enseñanza de español. Actas del L Congreso Internacional de la AEPE. La cultura hispánica: de sus orígenes al siglo XXi. Burgos: Universidad Isabel I de Castilla. Obtenido de https://cvc.cervantes.es/ensenanza/biblioteca_ele/aepe/pdf/congreso_50/congreso_50_09. pdf

Carrión, E. (2017). El uso de la Gamificación y los recursos digitales en el aprendizaje de las Ciencias Sociales en la Educación Superior. Revista DIM Didáctica, Innovacción y Multimedia(36). Obtenido de http://dimglobal.net/revistaDIM36/DIMOC36gamificacion.htm

Ciucci, L. A. (2016). Gamificación: alcances y perspectivas en la ciudad de La Plata. La Plata. Obtenido http://sedici.unlp.edu.ar/bitstream/handle/10915/57476/Documento_completo.6.3.pdfPDFA1b.pdf?sequence $=3 \&$ isAllowed $=y$

Foncubierta, J. M., \& Rodríguez, C. (2014). Didáctica de la gamificación en la clase de español. (8).

Obtenido

de https://espanolparainmigrantes.files.wordpress.com/2016/04/didactica_gamificacion_ele. pdf

Gallego, F., Molina, R., \& Llorens, F. (2014). Gamificar una propuesta docente Diseñando experiencias positivas de aprendizaje. XX Jornadas sobre la Enseñanza Universitaria de la informática, (pág. 2 ). Oviedo. Obtenido de https://rua.ua.es/dspace/bitstream/10045/39195/1/Gamificacio\%CC\%81n\%20(definicio\% CC\%81n).pdf

Gonzales, M. (2016). Gamificación. Hagamos que aprender sea divertido. Navarra: Universidad Pública de Navarra, España. Obtenido de https://academicae.unavarra.es/bitstream/handle/2454/21328/TFM15-MPES-\%20EGE-GONZALEZ68030.pdf? sequence $=1 \&$ isAllowed $=\mathrm{y}$

Hurtado, P., García, M., Rivera, D., \& Forgiony, J. (2018). Las estrategias de aprendizaje y la creatividad: una relación que favorece el procesamiento de la información. Revista Espacios, 39(17). Obtenido de http://www.revistaespacios.com/a18v39n17/18391712.html

López, N. J. (2016). Evaluación y TIC en primaria: el uso de Plickers para evaluar habilidades musicales. ENSAYOS, Revista de la Facultad de Educación de Albacete, 31(2), 81 - 90. Obtenido de https://revista.uclm.es/index.php/ensayos/article/view/1131

Marín, V. (2015). La gamificación educativa. Una alternativa para la enseñanza creativa. Digital Education Review(27). Obtenido de http://revistes.ub.edu/index.php/der/article/view/13433/pdf

Melo, M., \& Hernández, R. (2014). El juego y sus posibilidades en la enseñanza de las ciencias naturales. Innovación Educativa, 14(66). Obtenido de http://www.scielo.org.mx/scielo.php?script=sci_abstract\&pid=S166526732014000300004\&lng=es\&nrm=iso

Menendez Ferreira, R., Ruí1z Barquín, R., Maldonado, A., \& Camacho, D. (2018). Análisis y propuesta de una herramienta basada en gamificación para la educación en valores dentro del deporte. V Congreso de la Sociedad Española para las Ciencias del Videojuego, 1(1), 
1039-1045.

Obtenido

de https://sci2s.ugr.es/caepia18/proceedings/docs/CAEPIA2018_paper_245.pdf

Mesurado, B. (2010). La experiencia de Flow o Experiencia Óptima en el ámbito educativo. Revista Latinoamericana de Psicología, 42(2), 183-192. Obtenido de http://www.scielo.org.co/pdf/rlps/v42n2/v42n2a02.pdf

Mora, M., Calderón, M., \& Rubio, S. (2018). Kahoot, quizziz, plickers: una nueva forma de evaluar mediante la gamificación en las aulas 2.0. En P. d. educativos, Puebla, Belen; Rubira, Rainer; Magro, Silvia; Perea, Antonio; Nereida, Rubio; Sánchez, Inmaculada; Magalhães, María; Teixeira, María; López, Jesús; Vázquez, Teresa; Rodríguez, Tomás; Mora, Manuel; Calderón, Mónica; Rubio, Sebastián; Villena, Eduardo (Primera ed., pág. 135). Ediciones Egregius. Obtenido de https://drive.google.com/file/d/1Qh7yrMd51R9fGsQdpm8JSY9W1gzgd3ni/view

Morales, J. (2013). La gamificación en la universidad para mejorar los resultados académicos de los alumnos. Quinto Congreso Virtual Iberoamericano de Calidad en Educación Virtual y a Distancia, (págs. 1 - 15). México. Obtenido de http://eprints.uanl.mx/8087/1/m8_2.pdf

Muñoz, J., Muñoz, J., \& Ariza, T. (2017). Aplicación de juegos didácticos en el aula. La gamificación en la educación. Congreso Internacional sobre Innovación y Tendencias. Sevilla: INNTED.

Navarrete García, C. (2017). La gamificación al servicio de las Ciencias Sociales en Secundaria. Teoría y práctica docente. Madrid. Obtenido de http://eprints.ucm.es/46481/1/Navarrete_Garci\%CC\%81a_Claudia\%20_TFM.pdf

Novillo López, M. Á., Costa Román, Ó., Barrientos Fernández, A., Pericacho Gómez , F. J., Arigita García, A., \& Sánchez Cabrero, R. (2018). Gamificación: Un recurso para la motivación y la fidelización en los museos. CLIO. History and History teaching, 170-181. Obtenido de http://clio.rediris.es/n44/articulos/11Novillo2018.pdf

Ortiz Colón, A., Jordán, J., \& Agreda, M. (2018). Gamificación en educación: una panorámica sobre el estado de la cuestión. Revista Educ. Pesqu, 44, 1-17. Obtenido de http://www.scielo.br/pdf/ep/v44/1517-9702-ep-44-e173773.pdf

Parente, D. (2016). Gamificación en la educación. En R. Contreras Espinosa, \& J. L. Eguia, Gamificación en aulas universitarias (págs. 11-21). Barcelona: Universitat Autònoma de Barcelona. Obtenido de https://surassessment.cl/Documentos/publicaciones/Gamificaci\%C3\%B3n\%20en\%20aul as $\% 20$ univeristaria.pdf\#page $=11$

Parra, E., \& Torres, M. (2018). La gamificación como recurso didáctico en la enseñanza del diseño. Revista de investigación (EARI)(9), 160-173. Obtenido de https://dialnet.unirioja.es/servlet/articulo?codigo $=6994674$

Pérez Salazar, G. (2011). La Web 2.0 y la sociedad de la información. Revista Mexicana de Ciencias Políticas y Sociales, 56(212), 57-68. Obtenido de http://www.scielo.org.mx/pdf/rmcps/v56n212/v56n212a4.pdf

Pisonero, M. (2018). Monografías Sinoele. Gamificación en el aula de ELE: el avatar. IX Congreso Internacional de la Asociación Asiática de Hispanistas, Bangkok 2016, 17, págs. 326 324.

Bangkok.

Obtenido

de http://www.sinoele.org/images/Revista/17/monograficos/AAH_2016/AAH_2016_maria_ pisonero.pdf

Quero Virla, M. (2010). Confiabilidad y coeficiente Alpha de Cronbach. Telos, 12(2), 248-252. Obtenido de https://www.redalyc.org/pdf/993/99315569010.pdf 
Quesada, A. (2014). Videojuegos y educación. Procesos de aprendizaje, de creación y desarrollo de identidades virtuales. (El caso de World of Warcraft). 315. Universidad Autónoma de Barcelona, Departamento de Periodismo y Ciencias de la Comunicación. Obtenido de https://www.researchgate.net/publication/275027731_Videojuegos_y_educacion_Proceso s_de_aprendizaje_de_creacion_y_desarrollo_de_identidades_virtuales_El_caso_de_Worl d_of_Warcraft

Rodríguez, N., \& Elías, C. (2018). a gamificación aplicada al aprendizaje de la lengua española: Escape room en el aula de la ULPGC. V Jornadas Iberoamericanas de Innovación Educativa en el Ámbito de las TIC y las TAC, (págs. 179-183). Las Palmas de Gran Canaria. Obtenido de https://accedacris.ulpgc.es/handle/10553/52688

Román Sánchez, J. M., \& Gallego Rico, S. (2008). Acra Escalas de Estrategias de Aprendizaje. Madrid: TEA Ediciones, S.A. Obtenido de http://www.web.teaediciones.com/Ejemplos/ACRA_extracto_web.pdf

Romero Zegarra, F. (2016). Gamificación y tecnologías de información para el aprendizaje. Revista Experti, $\quad 1(2), \quad 20-24 . \quad$ Obtenido de http://revistas.upagu.edu.pe/index.php/EX/article/view/361

Torres Mesías, Á., Mora Guerrero, E., Garzón Velásquez, F., \& Ceballos Botina, N. E. (2013). Desarrollo de competencias científ cas a través de la aplicación de estrategias didácticas. Revista Tendencias, 14(1), 187-215. Obtenido de https://dialnet.unirioja.es/descarga/articulo/4453237.pdf

Torres, Á., \& Romero, L. (2018). Gamificación en Iberoamérica. Cuenca: Editorial Abya-Yala. Obtenido de https://idus.us.es/bitstream/handle/11441/79066/cap\%C3\%ADtulo\%20libro\%20Gamifica ci\%C3\%B3n\%20en\%20Iberoam\%C3\%A9rica.pdf?sequence=1\&isAllowed=y

Zambrano Izquierdo , D., Gomez Zermeño, M. G., \& Guerrero Roldan, A. E. (2016). Fundamentos teóricos de gamificación para un Sistema Tutorial Inteligente. Revista de Investigación Educativa de la Escuela de Graduados en Educación(13), 54-63. Obtenido de https://www.google.com/url?sa=t\&rct=j\&q=\&esrc=s\&source=web\&cd=1\&cad=rja\&uact $=8 \& v e d=2$ ahUKEwj8u4v3iazoAhWsSt8KHYueDV8QFjAAegQIBBAB\&url=https\%3A $\% 2 \mathrm{~F} \% 2 \mathrm{Fwww}$.rieege.mx\%2Findex.php\%2Frieege $\% 2$ Farticle $\% 2 F d o w n l o a d \% 2 F 420 \% 2 \mathrm{~F}$ 250\&usg=AOvVaw1 gpTOx-hdGat7yxkq3085c 


\section{Anexo 1}

\section{Cuestionario de Satisfacción de la técnica de gamificación}

Dirigido: Estudiantes de noveno año de educación básica Institución: Escuela de Educación Básica Naciones Unidas

Estimado estudiante, con el fin de determinar el grado de satisfacción del uso de la técnica de gamificación en el aprendizaje de las ciencias naturales, solicito llene el siguiente cuestionario en donde los significados de cada una de las letras son: A (extremadamente satisfactorio), B (muy satisfactorio), C (moderadamente satisfactorio), D (poco satisfactorio), e (nada satisfactorio), tachar una respuesta en cada ítem. El mismo que es completamente anónimo.

1.- ¿Le pareció adecuada la utilización de la gamificación en la unidad tratada?
A B
$\mathrm{C}$
$\mathrm{D}$
$\mathrm{E}$

2.- Se sintió motivado al realizar los deberes con la técnica de gamificación

A $\quad$ B $\quad$ C $\quad$ D $\quad$ E

3.- ¿Cree que existió mayor interacción y entretenimiento al desarrollar la técnica de gamificación en las clases de ciencias naturales?

A $\quad$ B $\quad$ C $\quad$ D $\quad$ E

4.- ¿Cree que su aprendizaje mejoró con la utilización de técnicas de gamificación?

A $\quad$ B $\quad$ C $\quad$ D $\quad$ E

5.- ¿Le pareció interactiva las clases de ciencias naturales con el uso de estas técnicas de gamificación?

A $\quad$ B $\quad C \quad$ D $\quad E$

6.- ¿Te pareció novedoso el uso la técnica de gamificación en el aprendizaje de ciencias naturales?

A $\quad$ B $\quad$ C $\quad$ D $\quad$ E

7.- ¿Crees que sería una mejor forma de aprender ciencias naturales, al utilizar técnicas de gamificación?

A $\quad$ B $\quad$ C $\quad$ D $\quad$ E

8.- ¿Le gustó trabajar en equipos y ganar puntos por competición?

A $\quad$ B $\quad$ C $\quad$ D $\quad$ E

9- ¿Considera usted que ha adquirido una mayor comprensión y retención de conocimientos en comparación con las clases sin gamificación?

A $\quad$ B $\quad$ C $\quad$ D $\quad$ E

10.- ¿Cuál herramienta de gamificación le pareció más atractiva e interactiva para su aprendizaje?

Kahoot

Plickers 